\title{
Research Paper: Comparison of Memory Impairment and Oxidative Stress Following Single or Repeated Doses Administration of Scopolamine in Rat Hippocampus
}

\author{
Milad Rahimzadegan ${ }^{1}$, Maliheh Soodi ${ }^{1 *}$
}

1. Department of Toxicology, School of Medical Sciences, Tarbiat Modares University, Tehran, Iran

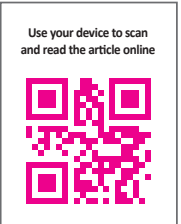

Citation: Rahimzadegan, M., \& Soodi, M. (2018). Comparison of Memory Impairment and Oxidative Stress Following Single or Repeated Doses Administration of Scopolamine in Rat Hippocampus. Basic and Clinical Neuroscience, 9(1), 5-14. https://doi.org/10.29252/NIRP.BCN.9.1.5

: https://doi.org/10.29252/NIRP.BCN.9.1.5

Article info:

Received: 02 May 2016

First Revision: 14 June 2016

Accepted: 25 February 2017
Key Words:

Scopolamine,

Oxidative stress,

Acetylcholinesterase,

Memory

\begin{abstract}
A B S T RA C T
Introduction: Scopolamine, a muscarinic cholinergic receptor antagonist, is widely used to induce memory impairment in experimental animals. The present study aims to compare memory impairment and oxidative stress following single and repeated doses administration of scopolamine.

Methods: A group of rats received a single shot of scopolamine in different doses $(0.5,1$, or 3 $\mathrm{mg} / \mathrm{kg}$, IP) 24 hours after the passive avoidance training. Then the memory retrieval test was performed 30 minutes and 7 days after the injection. In the other experiment, rats received similar doses of scopolamine for 7 consecutive days, 24 hours after the training session. Then the memory retrieval test was performed 30 minutes and 7 days after the last injection. Acetylcholinesterase $(\mathrm{AChE})$ activity and lipid peroxidation were measured in their hippocampus tissue, too.

Results: Scopolamine administered in repeated doses caused more impairment in memory function compared to single dose injection based on the evaluation 30 minutes after injection. Moreover, the memory impairment persisted for 7 days only in repeated doses treated groups. Increase in acetylcholinesterase activity and lipid peroxidation in both groups was observed 30 minutes after scopolamine administration. These abnormal increases persisted for 7 days only in repeated doses treated groups. Increased AChE activity and lipid peroxidation was well correlated with behavioral deficit. Also AChE activity was well associated with lipid peroxidation.

Conclusion: The results of present study showed that repeated administration of scopolamine induced results in memory impairment. This effect can be due to long-lasting oxidative stress which may damage the hippocampus tissue.
\end{abstract}

\section{Introduction}

copolamine, a tropane alkaloid isolated from plant sources, is a nonselective muscarinic antagonist, which induces memory impairment (Klinkenberg \& Blokland,
2010). Central cholinergic system plays a crucial role in learning and memory functions for humans and animals. According to the cholinergic hypothesis, elderly memory dysfunction is due to loss of central cholinergic neurons and decreased levels of brain acetylcholine (Perry, 1986). Scopolamine-induced amnesia,

* Corresponding Author:

Maliheh Soodi, PhD

Address: Department of Toxicology, School of Medical Sciences, Tarbiat Modares University, Tehran, Iran.

Tel:+98 (21) 82884549

E-mail:soodi@modares.ac.ir 
most likely caused by a blockade of cholinergic signaling, is used as a pharmacological model of Alzheimer Disease (AD) (Ebert \& Kirch, 1998). Various behavioral studies have been carried out to investigate the effect of scopolamine on learning and memory. In this regard, administration of scopolamine induces performance deficits in the radial maze (Burešová \& Bureš, 1982). Peripheral administration of scopolamine can constantly interfere with short-term memory in object recognition and spatial alternation tasks (Sambeth, Riedel, Smits, \& Blokland, 2007). Also scopolamine impairs memory acquisition and retention in Morris water and passive avoidance (Cozzolino et al., 1994).

It is postulated that blockade of muscarinic receptors is the main mechanism of scopolamine-induced memory impairment, but several studies argued that stimulation or blockade of other neurotransmitter systems such as glutamatergic (Gutierres et al., 2014; Mahmoodi, Ahmadi, Oryan, \& Zarrindast, 2010), adrenergic (Azami et al., 2010), dopaminergic (Aalto, 2005; Dash, Moore, Kobori, \& Runyan, 2007), serotonergic (Barnes \& Sharp, 1999; Da Silva Costa-Aze, Quiedeville, Boulouard, \& Dauphin, 2012; Dash, et al., 2007), and histaminergic systems (Chen \& Kamei, 2000) could ameliorate scopolamine-induced memory impairment suggesting that these neurotransmitters may be involved in scopolamineinduced memory impairment. Also scopolamine induces oxidative stress (Jang et al., 2013), apoptosis (Jahanshahi, Nickmahzar, \& Babakordi, 2013), and inflammatory responses (Ahmad, Ramasamy, Jaafar, Majeed, \& Mani, 2014) in the brain tissue. So the exact mechanism of scopolamine is still unknown.

Several studies have reported that oxidative stress is one of the mechanisms involved in scopolamine-induced amnesia. Scopolamine decreases the activity of superoxide dismutase, catalase, glutathione s-transferase, and glutathione peroxidase (Uma \& Maheswari, 2014), also it increase the level of malondialdehyde marker of lipid peroxidation (Abd-El-Fattah, Abdelakader, \& Zaki, 2014). Several antioxidant compounds could ameliorate scopolamine-induced memory impairment by attenuating oxidative stress markers (Harrison, Hosseini, Dawes, Weaver, \& May, 2009; Hritcu, Stefan, Brandsch, \& Mihasan, 2015) indicating crucial role of oxidative stress in scopolamine-induced amnesia.

Numerous studies have used scopolamine to induce memory impairment in experimental animals but the doses of scopolamine administration were different in these studies. Some used single dose and some repeated doses of scopolamine. Thus, it is not clear weather intensity and duration of scopolamineinduced amnesia differ following single or repeated doses. Because of the complex action of scopolamine it seems that mechanisms of scopolamine-induced amnesia are different following administration of single dose or repeated doses. As scopolamine is used as the reference standard drug for inducing memory impairment in experimental animals also the animal model of scopolamine is used for preclinical study of new substances in treatment of dementia, better understanding of scopolamine-induced memory impairment helps researchers better interpret the results. The present study aims to compare memory impairment and oxidative stress after administration of single or repeated doses of scopolamine.

\section{Methods}

\subsection{Animals}

Male Wistar rats were maintained in constant temperature $\left(25^{\circ} \mathrm{C}\right)$ and $12: 12 \mathrm{~h}$ light/dark cycle and fed with a standard laboratory diet and water. All animal experiments were approved by Ethics Committee of the Tarbiat Modares University.

\subsection{Study materials}

Scopolamine hydrobromide, Acetylthiocholin (ATCh), 5,5-dithiobis (2-nitrobenzioc) acid (DTNB), Thiobarbituric Acid (TBA), malondialdehyde bis (dimethyl acetal) were purchased from Sigma (USA).

\subsection{Experimental design}

The experimental design included two experiments: Experiment 1; and Experiment 2. In experiment 1, the effect of single dose administration of scopolamine on memory retrieval was assessed. The animals were trained in passive avoidance apparatus. About 24 hours after training, the animals received saline or different doses of scopolamine intraperitoneally $(0.5,1$, and $3 \mathrm{mg} /$ $\mathrm{kg}$ ) and their memory retrieval was assessed 30 minutes and 7 days after the injection.

In experiment 2, the effect of administration of scopolamine in repeated doses on memory retrieval was assessed. The animals were trained in passive avoidance apparatus. About 24 hours after training, the animals received saline or different doses of scopolamine intraperitoneally $(0.5,1$, or $3 \mathrm{mg} / \mathrm{kg})$ for seven consecutive days and then their memory retrieval was assessed 30 minutes and 7 days after the last injection. 


\subsection{Passive avoidance test}

The step-through inhibitory avoidance apparatus consisted of two compartments of the same size $(20 \times 20 \times 30$ $\mathrm{cm})$ separated by a guillotine door $(7 \times 9 \mathrm{~cm})$. The walls and floor of one compartment are made of white opaque resin which forms light compartment. The walls of the other compartment are black and forms dark compartment. The floor of the dark compartment is made of stainless steel bars ( $3 \mathrm{~mm}$ in diameter and $1 \mathrm{~cm}$ apart). Intermittent electric shocks $(50 \mathrm{~Hz}$ for $3 \mathrm{~s}$, and with 1 $\mathrm{mA}$ intensity) were passed on to the grid floor of the dark compartment by an insulated stimulator.

All rats were placed in the experimental room and allowed to habituate for at least $30 \mathrm{~min}$ before the experiments. Then each rat was kindly located in the light compartment of the apparatus. The guillotine door was opened after $5 \mathrm{~s}$ and the animal was allowed to enter the dark compartment. The time taken for the animal to enter the dark compartment with all four-paws ( $\mathrm{Re}$ tention time) is recorded. All rats stayed more than 120 $\mathrm{s}$ in light chamber and did not enter the dark compartment were excluded from the experiments. Once the rat entered with all four-paws to the dark part, the guillotine door was closed and an electrical foot shock (50 $\mathrm{Hz}, 1 \mathrm{~mA}$ for $3 \mathrm{~s}$ ) was delivered through the stainless steel bars. The rat was removed from dark compartment after foot shock and trained again. The training was terminated after the rat remained in the light compartment for $120 \mathrm{~s}$. The number of trials (entries into the dark chamber) was recorded. All the rats were trained with a maximum of 3 trials.

A retrieval test was carried out to determine the longterm memory formation $24 \mathrm{~h}$ and 7 days after the training. Each animal was placed in the light compartment for $20 \mathrm{~s}$, then the door was opened and the step-through latency for entering the dark compartment (retention time) was measured. The cut-off time of $300 \mathrm{~s}$ was applied for those animals which still remained in the light compartment. During these sessions, no electric shock was applied.

\subsection{Open field}

The open field apparatus was made of wooden arena, $40 \times 40 \mathrm{~cm}$, enclosed by a wall of $25 \mathrm{~cm}$ height. The lines were drawn on the floor with a marker which divided the arena into nine equal areas (with $3 \times 3$ divisions). After administration of the highest dose of scopolamine ( 3 $\mathrm{mg} / \mathrm{kg}$ ), for seven consecutive days, each rat was placed in the center of platform. Activity was recorded for 15 minutes and speed parameter was measured with video tracking software (Ethovision XT, Nuldos, Netherlands).

\subsection{Preparation of brain tissue samples for bio- chemical analysis}

After training, the animals were killed by decapitation under ether anesthesia, and their brain tissues were removed and washed with ice cold $0.1 \mathrm{M}$ phosphate buffer saline $(\mathrm{pH}=7.4)$ then the their hippocampuses were dissected out and homogenized in ice cold PBS with $1 \%$ Triton $\mathrm{X}$. The homogenates were then centrifuged at $3000 \times \mathrm{g}$ for $10 \mathrm{~min}$ at $4^{\circ} \mathrm{C}$, and the supernatant was used.

\subsection{Measurement of brain AChE activity}

Brain acetylcholinesterase activity was measured according to Ellman method (Ellman, Courtney, Andres, \& Feather-Stone, 1961). To prepare Ellman reagent, $0.1 \mathrm{M}$ phosphate buffer ( $\mathrm{pH}=8.0)$, acetylthiocholin iodide (ATCh, $75 \mathrm{mM}$ ) as a substrate, and 5, 5-dithiobis (2-nitrobenzioc) acid (DTNB, $10 \mathrm{mM}$ ) were mixed in a ratio of 150:2:5. Then, $140 \mu \mathrm{L}$ of Ellman reagent was transferred to the each well of 96 well plate. About 10 $\mu \mathrm{L}$ of brain homogenate as an enzyme source was added to Ellman reagent in the plate and the absorbance was immediately monitored during $6 \mathrm{~min}$ at $405 \mathrm{~nm}$ using a microplate reader (BIO TEK). The activity was calculated based on extinction coefficient of Thionitrobenzoation (TNB). Protein concentration in the brain homogenate was measured using the Bradford method (Bradford, 1976).

\subsection{Measurement of lipid peroxidation}

Malondialdehyde (MDA) was measured as an index of lipid peroxidation level in hippocampal tissue. The Thiobarbituric Acid Reaction (TBAR) colorimetric assay was used for the measurement of MDA level (Soodi et al., 2015). TBA reagent consisted of $3.75 \%$ TCA and $0.0925 \%$ TBA. Two volumes of TBA reagent and one volume of brain homogenate were mixed in a microtube and the mixture was incubated at $90^{\circ} \mathrm{C}$ for $60 \mathrm{~min}$. After incubation the microtube was placed on ice to cooling. After cooling, the microtube was centrifuged at $1000 \mathrm{xg}$ for $10 \mathrm{~min}$ and optical density of supernatant was measured in $540 \mathrm{~nm}$ in plate reader. MDA standard curve was established with using the stable MDA precursor, malondialdehyde bis (dimethyl acetal).

\subsection{Statistical analysis}

The results were expressed as mean $\pm \mathrm{SEM}$. The statistical differences were analyzed by 2-way analysis of 
variances (ANOVA) followed by Bonferroni multiple comparisons test. The correlation between study data was analyzed by the Pearson test. The probability level less than 0.05 were considered as significant.

\section{Results}

3.1. Comparison of the single and repeated doses administration of scopolamine effects on memory retrieval

In this study, the effect of single and repeated dose administration of scopolamine on retrieval of inhibitory avoidance memory was investigated. Twenty-four hours after training, scopolamine $(0.5,1$, and $3 \mathrm{mg} / \mathrm{kg})$ was injected intraperitoneally and memory retrieval was assessed 30 minutes after injection. Figure 1A shows the results of the memory retrieval test. Significant dose effect was observed in treated groups, $\mathrm{F}_{(3,35)}=40.09$, $\mathrm{P}<0.001$. Single dose scopolamine dose-dependently caused memory loss, also memory impairment was observed in repeated doses treated group but this effect was not dose dependent and significant differences were not observed between different doses of scopolamine. However, significant differences were observed between effect of single dose and repeated doses of scopolamine on the memory retrieval test and repeated doses of scopolamine impair memory more potent than single dose, $\mathrm{F}_{(1,35)}=26.68, \mathrm{P}<0.001$

Figure 1B shows the effects of scopolamine single dose and repeated doses on memory retrieval test seven days after injection. A significant effect was observed in repeated doses treated groups $\mathrm{F}_{(3,36)}=11.23, \mathrm{P}<0.001$. In single dose treated group there is no significant differences between control and treated groups and memory retrieval was not affected by single dose administration of scopolamine after seven days. In repeated doses treated groups, memory retrieval significantly was affected by scopolamine treatment and retention time significantly decreased in repeated doses treated groups compared to the single dose treated groups $\mathrm{F}_{(1,36)}=105.6, \mathrm{P}<0.001$.

\subsection{Effect of scopolamine on locomotor activity}

For evaluating the effect of scopolamine on locomotor activity, open field test was performed on animals which received the high dose of scopolamine $(3 \mathrm{mg} / \mathrm{kg}$ ) for 7 days. Results indicated that locomotor activity was not significantly different between scopolamine-treated and saline-treated groups (Figure 2).

\subsection{Comparison of single and repeated doses ad- ministration of scopolamine on $\mathrm{AChE}$ activity}

Results of AChE activity in the hippocampus are shown in Figure 3. Both single dose and repeated doses administration of scopolamine significantly increased AChE activity compared to control group 30 min after injections $\mathrm{F}_{3 \text {, }}$ 36) $=165.9, \mathrm{P}<0.001$. Statistical analysis indicated that $\mathrm{AChE}$ activity more increased in repeated doses groups than single dose groups $\mathrm{F}_{(1,36)}=70.17, \mathrm{P}<0.001$ (Figures $3 \mathrm{~A}$ ). Seven days after injection, increase in $\mathrm{AChE}$ activity was observed in repeated doses treated groups but not in the single dose treated groups $\mathrm{F}_{(1,36)}=66.95, \mathrm{P}<0.001$ (Figures $3 \mathrm{~B}$ ).

3.4. Comparison of single and repeated doses administration of scopolamine effects on lipid peroxidation

The Thiobarbituric Acid Reactive Substances (TBARS) are widely considered as the most common assay to measure the lipid peroxidation. Scopolamine dose-dependently increased the level of MDA in both single and repeated doses treated groups $30 \mathrm{~min}$ after in-

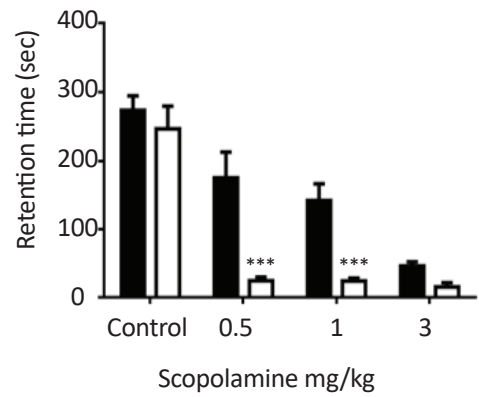

Single does $\square$ Repeated does

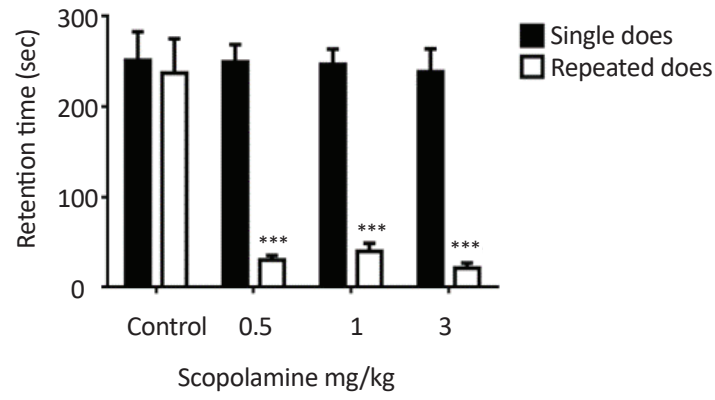

NEUR SCIENCE

Figure 1. The effects of single and repeated doses administration of scopolamine on memory retrieval

Rats intraperitoneally received different doses of scopolamine $24 \mathrm{~h}$ after training as a single dose or for seven consecutive days and retrieval test was performed 30 min after injection (A) or seven days after injection (B). 


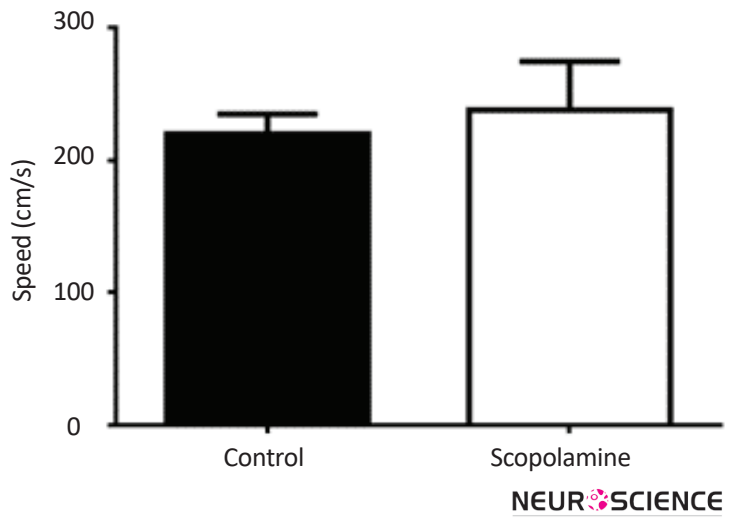

Figure 2. Locomotor activity after scopolamine treatment

Scopolamine $(3 \mathrm{mg} / \mathrm{kg})$ was administrated for seven consecutive days and locomotor activity measured by open field apparatus.

jection $\mathrm{F}_{(3,36)}=76.25, \mathrm{P}<0.001$ but the level of MDA was more in repeated doses treated groups than single dose treated groups $\mathrm{F}_{(1,36)}=116.8, \mathrm{P}<0.001$ (Figure 4A). In single dose treated groups there is no difference in MDA level between treated and control groups seven days after injection but in repeated doses treated groups significant increase in the level of MDA was observed seven days after last injection $\mathrm{F}_{(1,36)}=117.5, \mathrm{P}<0.001$ (Figure 4B).

\subsection{Correlation between biochemical parameters} and behavioral results

Significant correlation between retention time and $\mathrm{AChE}$ activity and also between retention time and lipid peroxidation were observed in all groups except single dose treated group tested seven days after injection (Figures 5 and 6). Also significant correlation was observed between AChE activity and lipid peroxidation (Figure 7).

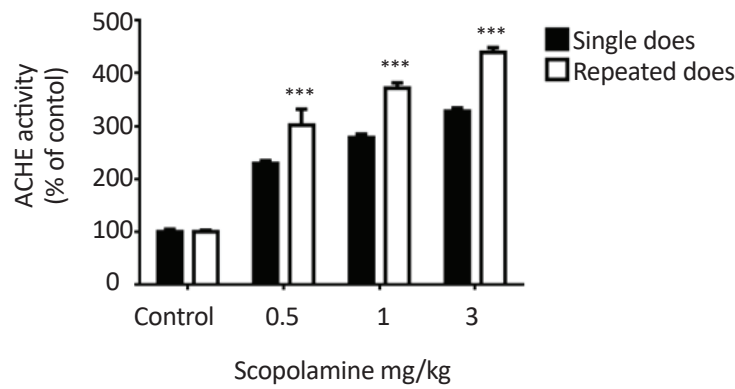

\section{Discussion}

In the present study, effects of single dose and repeated doses administration of scopolamine on memory retrieval and brain biochemical parameters were compared. Scopolamine is widely used for induction of memory impairment in experimental animals and scopolamineinduced amnesia is used as a model for studying therapeutic agents proposed to use in $\mathrm{AD}$ therapy. To this end, most studies have used single administration of scopolamine which induces reversible memory impairment and some studies have used multiple administration of scopolamine, especially studies which applied Morris water maze for assessing memory performance. But there is no report about the differences in the memory impairment, its stability and also biochemical changes in the hippocampus following repeated doses and single dose scopolamine administration which was the main purpose of this study. Results of the behavioral tests indicated that single dose administration of scopolamine dose-dependently impaired memory retrieval $30 \mathrm{~min}$ after injection but did not affect memory retrieval seven days after injection. These results suggest that single dose scopolamine induces reversible amnesia. Also results indicate that repeated doses administration of scopolamine produce memory impairment more potent than single dose and this effect is stable even seven days after last injection. Based on these results, we can conclude that different mechanisms may be involved in memory impairment after single dose and repeated doses administration of scopolamine.

Blockade of muscarinic receptor is the main mechanism for the scopolamine-induced memory impairment; however, the involvement of other receptors has been reported, too. Chronic administration of scopolamine increases expression of M1 muscarinic and $\alpha 7$ nicotinic acetylcho-

B

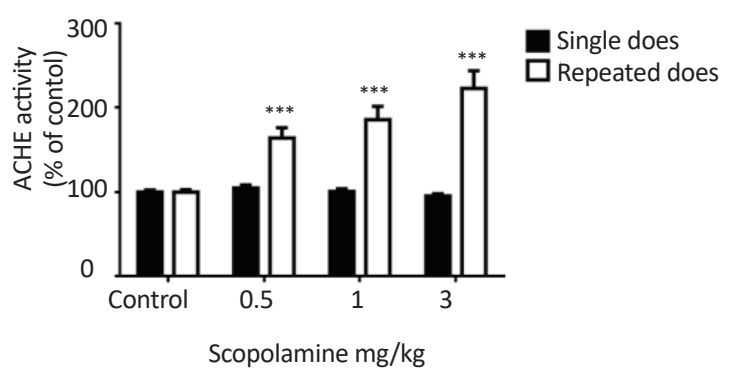

NEUR:SCIENCE

Figure 3. The effects of single and repeated doses administration of scopolamine on hippocampus AChE activity

Rats intraperitoneally received different doses of scopolamine $24 \mathrm{~h}$ after training as a single dose or for seven consecutive days and retrieval test was performed 30 min after injection (A) or seven days after injection (B).

*** $\mathrm{P}<0.001$ represents significant differences from single dose. 

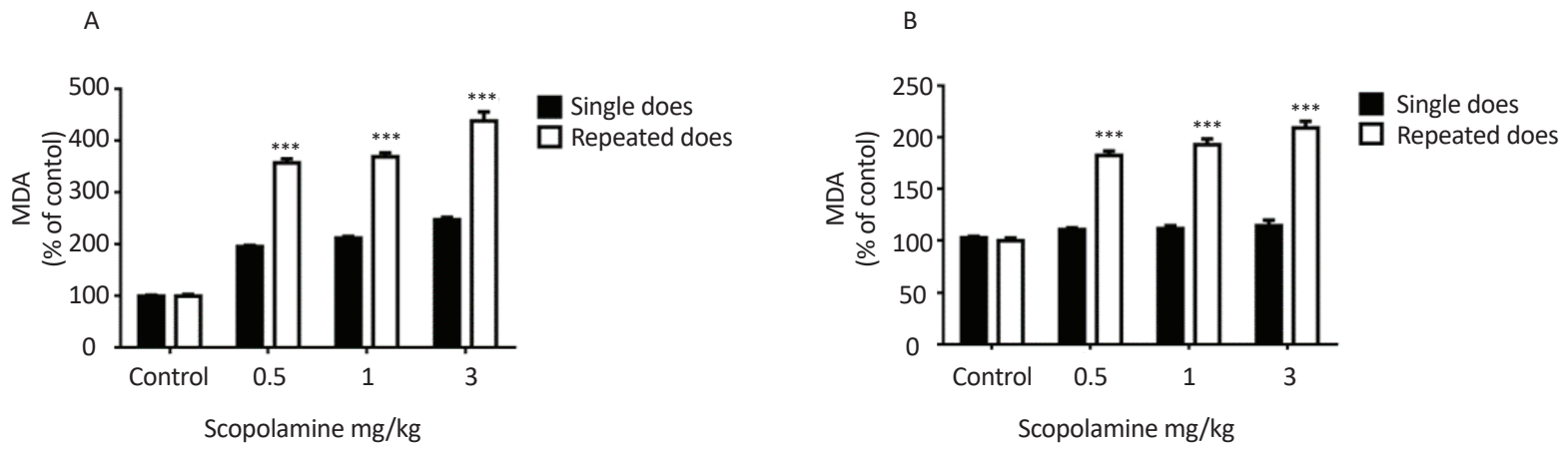

Figure 4. The effects of single and repeated doses administration of scopolamine on hippocampus lipid peroxidation

Rats intraperitoneally received different doses of scopolamine $24 \mathrm{~h}$ after training as a single dose or for seven consecutive days and retrieval test was performed $30 \mathrm{~min}$ after injection (A) or seven days after injection (B).

*** $\mathrm{P}<0.001$ represents significant differences from single dose.

line receptors and NR1 subunit of glutamate receptors in the hippocampus (Falsafi, Deli, Höger, Pollak, \& Lubec, 2012). These receptors are co-localized in the glutamatergic synapse in the hippocampus and have crucial roles in memory formation (Marino, Rouse, Levey, Potter, \& Conn, 1998). Then it is suggested that memory impairment after repeated doses administration of scopolamine is not only due to blockade of muscarinic receptor alone and changes in acetylcholine and glutamate receptors subunit expression pattern are involved in prolong memory impairment by repeated doses scopolamine administration.

Several studies reported that scopolamine-induced memory impairment is associated with increased oxi-
A

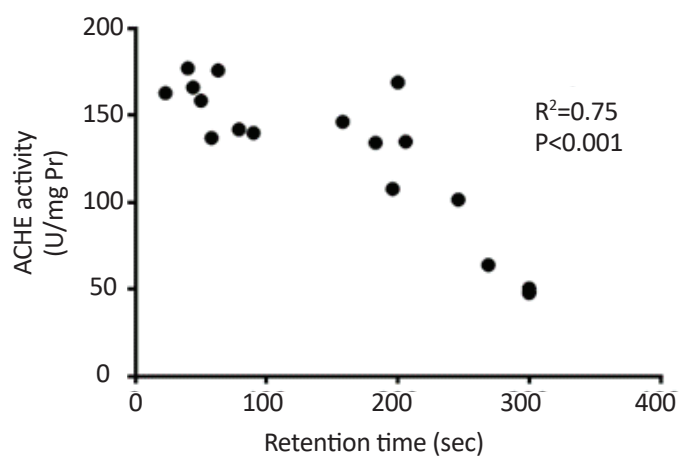

C

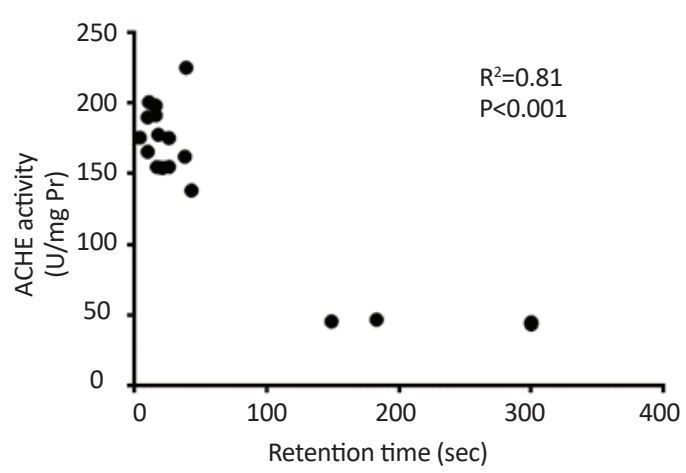

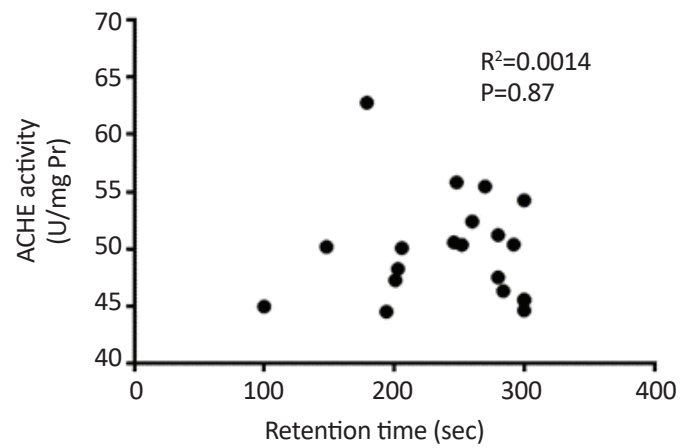

D

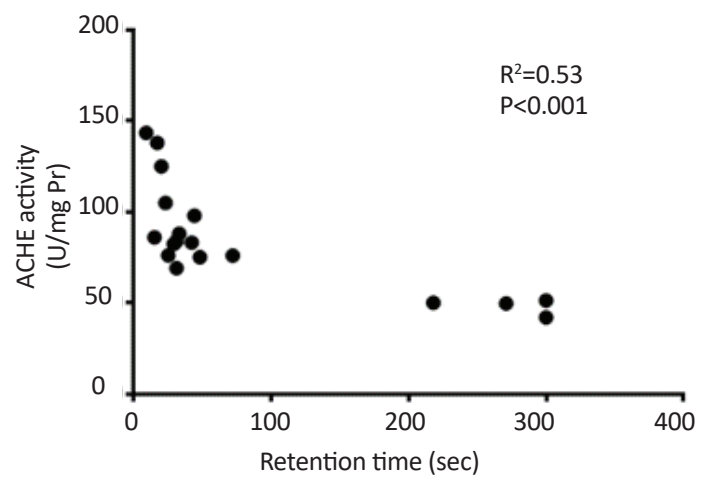

Figure 5. Correlation between AChE activity and behavioral test

NEUR:SCIENCE

A: Single dose scopolamine administered and retrieval test was performed after $30 \mathrm{~min}$; B: Single dose scopolamine administered and retrieval test was performed after 7 days; C: Scopolamine administered for 7 consecutive days and retrieval test was performed after $30 \mathrm{~min}$; D: Scopolamine administered for 7 consecutive days and retrieval test was performed after 7 days. 


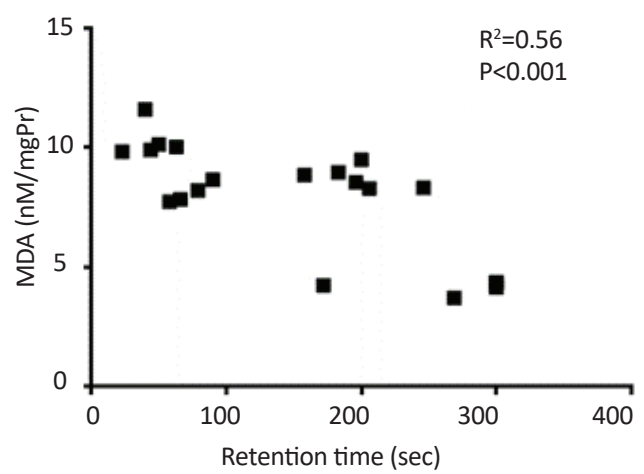

C

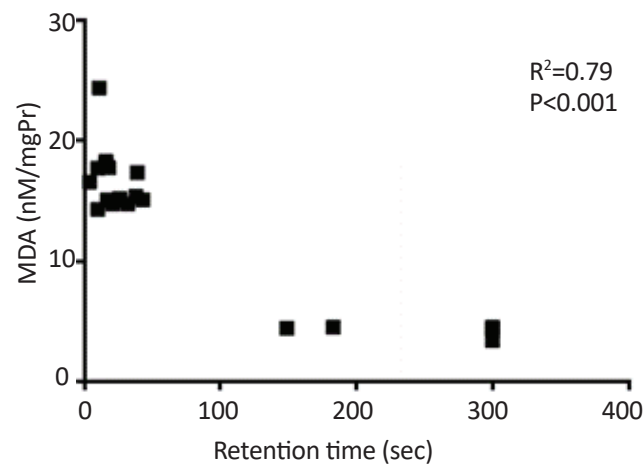

B

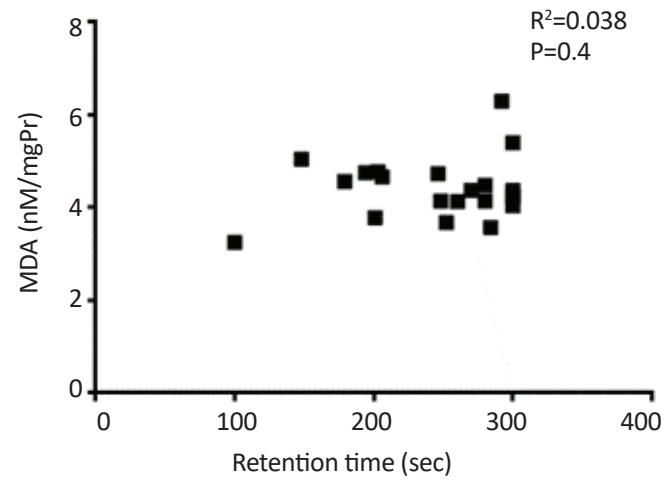

D

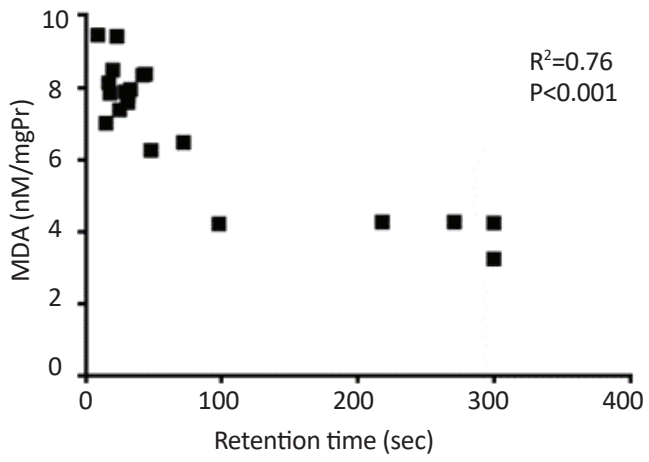

NEUR:SCIENCE

Figure 6. Correlation between lipid peroxidation and behavioral test

A: Single dose scopolamine administered and retrieval test was performed after $30 \mathrm{~min}$; B: Single dose scopolamine administered and retrieval test was performed after 7 day; C: Scopolamine administered for 7 consecutive days and retrieval test was performed after $30 \mathrm{~min}$; D: Scopolamine administered for 7 consecutive days and retrieval test was performed after 7 days.

dative stress within the brain (Fan et al., 2005). In the present study, significant increase in the brain level of malondialdehyde, which is the measure of lipid peroxidation, was observed $30 \mathrm{~min}$ after scopolamine treatment. The same result was achieved in repeated dose administration but lipid peroxidation was much more than single injection group and also lipid peroxidation was continued for 7 days in repeated dose group but significant lipid peroxidation was not observed in single dose groups 7 days after injection. These results indicate that single dose scopolamine produces reversible lipid peroxidation but repeated doses induce stable lipid peroxidation which persists at least one week. In our experiments, lipid peroxidation was well correlated with behavior deficit. Several studies have suggested that increased inflammation and oxidative stress is associated with cognitive deficits. Inflammation plays an important role in neurodegenerative disorders (Mhatre, Floyd, \& Hensley, 2004).
Increased level of pro-inflammatory cytokines including TNF- $\alpha$, Interleukin (IL)-1, and IL-6 are shown in the brains of patients with dementia (Akiyama et al., 2000; Fillit et al., 1991; Hauss-Wegrzyniak, Lynch, Vraniak, \& Wenk, 2002). It has been reported that chronic administration of scopolamine for 14 days increased the expression of pro-inflammatory factors such as IL-1 $\beta$ and $\mathrm{TNF} \alpha$ and inflammation is a proposed mechanism for scopolamine-induced memory impairment (Ahmad et al., 2014). It is suggested that blockage of muscarinic receptor by scopolamine might increase the expression of TNF- $\alpha$ in the hippocampus (Jang et al., 2013). Inflammation is associated with generation of oxygen free radicals and oxidative stress. Then it is proposed that repeated doses administration of scopolamine induces chronic inflammation and oxidative stress which resulted in prolong memory impairment.

Several studies reported that AChE activity increases after scopolamine injection (Soodi, Naghdi, Hajimeh- 
A

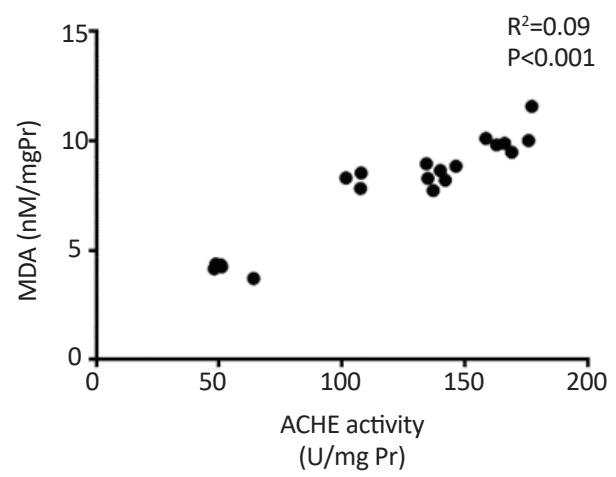

C

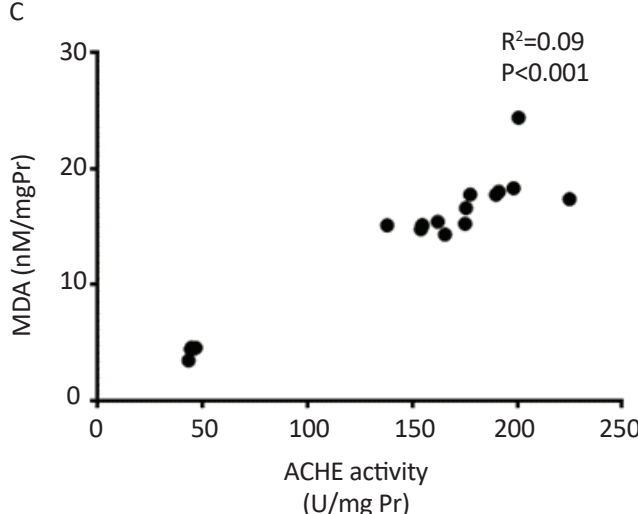

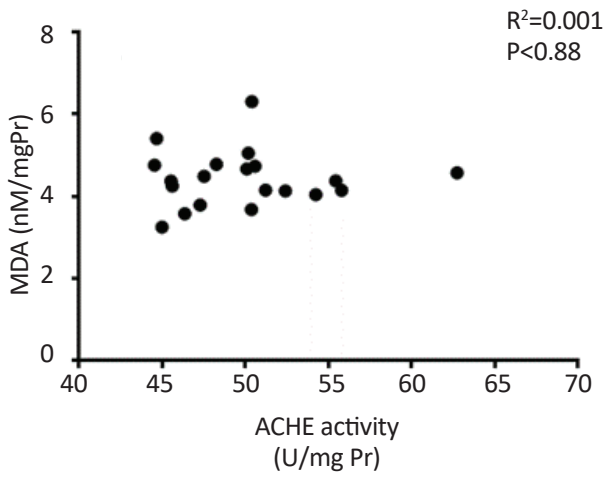

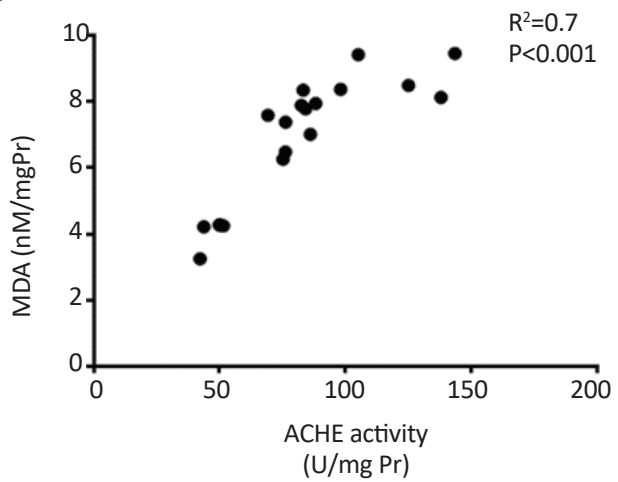

NEUR SIENCE

Figure 7. Correlation between AChE activity and lipid peroxidation

A: Single dose scopolamine administered and retrieval test was performed after $30 \mathrm{~min}$; B: Single dose scopolamine administered and retrieval test was performed after 7 days; C: Scopolamine administered for 7 consecutive days and retrieval test was performed after $30 \mathrm{~min}$; D: Scopolamine administered for 7 consecutive days and retrieval test was performed after 7 days.

dipoor, Choopani, \& Sahraei, 2014). Increased level of AChE metabolizes more acetylcholine and reduces its level in the synapses then weakens cholinergic neurotransmission which resulted in memory impairment. Studies indicate that AChE inhibitors can reverse scopolamine-induced memory impairment (Chaudhaery et al., 2010). The mechanism of AChE activity increase by scopolamine is not completely known yet. Other than the catalytic activity of acetylcholinesterase, the noncatalytic activities were assumed for acetylcholinesterase protein. Some evidence suggests that expression of this protein was induced in stress condition such as oxidative stress (Härtl, Gleinich, \& Zimmermann, 2011). Then it is postulated that scopolamine-induced oxidative stress is a possible mechanism of $\mathrm{AChE}$ activity increase and subsequent memory impairment. In our study, AChE activity increase was well correlated with memory deficit. Also similar to lipid peroxidation marker, increased level of AChE activity was more predominant in repeated dose group and persist at least seven days after last injec- tion and increase in $\mathrm{AChE}$ activity well correlated with lipid peroxidation.

Our study indicated that repeated doses of scopolamine administration impair memory function worse and more prolong than single dose and chronic oxidative stress may be the possible mechanism. Because chronic inflammation and oxidative stress have been observed in the brain of $\mathrm{AD}$ patient it seems that administration of repeated doses of scopolamine better simulate the AD condition and it is recommended that chronic administration of scopolamine is used for preclinical testing of new substances designed to treat $\mathrm{AD}$.

\section{Acknowledgements}

This research was extracted from the first author's MSc. thesis in the Department of Toxicology, School of Medical Sciences, Tarbiat Modares University of Tehran and was also financially supported by Tarbiat Modares University. 


\section{Conflict of Interest}

The authors declared no conflict of interest.

\section{References}

Aalto, S. (2005). Frontal and temporal dopamine release during working memory and attention tasks in healthy humans: A positron emission tomography study using the high-affinity dopamine D2 receptor ligand [11C]FLB 457. Journal of Neuroscience, 25(10), 2471-7. doi: 10.1523/jneurosci.2097-04.2005

Abd-El-Fattah, M. A., Abdelakader, N. F., \& Zaki, H. F. (2014). Pyrrolidine dithiocarbamate protects against scopolamine-induced cognitive impairment in rats. European Journal of Pharmacology, 723, 330-8. doi: 10.1016/j.ejphar.2013.11.008

Ahmad, A., Ramasamy, K., Jaafar, S. M., Majeed, A. B. A., \& Mani, V. (2014). Total isoflavones from soybean and tempeh reversed scopolamine-induced amnesia, improved cholinergic activities and reduced neuroinflammation in brain. Food and Chemical Toxicology, 65, 120-8. doi:10.1016/j.fct.2013.12.025

Akiyama, H., Arai, T., Kondo, H., Tanno, E., Haga, C., \& Ikeda, K. (2000). Cell mediators of inflammation in the alzheimer disease brain. Alzheimer Disease and Associated Disorders, 14(Supplement), S47-S53. doi: 10.1097/00002093-200000001-00008

Azami, N. S., Piri, M., Oryan, S., Jahanshahi, M., Babapour, V., \& Zarrindast, M. R. (2010). Involvement of dorsal hippocampal a-adrenergic receptors in the effect of scopolamine on memory retrieval in inhibitory avoidance task. Neurobiology of Learning and Memory, 93(4), 455-62. doi: 10.1016/j.nlm.2010.01.003

Barnes, N. M., \& Sharp, T. (1999). A review of central 5-HT receptors and their function. Neuropharmacology, 38(8), 1083-152. doi: 10.1016/s0028-3908(99)00010-6

Bradford, M. (1976). A rapid and sensitive method for the quantitation of microgram quantities of protein utilizing the principle of protein-dye binding. Analytical Biochemistry, 72(1-2), 248-54. doi: 10.1006/abio.1976.9999

Burešová, O., \& Bureš, J. (1982). Radial maze as a tool for assessing the effect of drugs on the working memory of rats. Psychopharmacology, 77(3), 268-71. doi: 10.1007/bf00464578

Chaudhaery, S. S., Roy, K. K., Shakya, N., Saxena, G., Sammi, S. R., Nazir, A., et al. (2010). Novel carbamates as orally active acetylcholinesterase inhibitors found to improve scopolamine-induced cognition impairment: Pharmacophore-based virtual screening, synthesis, and pharmacologyt. Journal of Medicinal Chemistry, 53(17), 6490-505. doi: 10.1021/jm100573q

Chen, Z., \& Kamei, C. (2000). Facilitating effects of histamine on spatial memory deficit induced by scopolamine in rats. Acta pharmacologica Sinica, 21(9), 814-8. PMID: 11501163

Cozzolino, R., Guaraldi, D., Giuliani, A., Ghirardi, O., Ramacci, M. T., \& Angelucci, L. (1994). Effects of concomitant nicotinic and muscarinic blockade on spatial memory disturbance in rats are purely additive: Evidence from the morris water task. Physiology \& Behavior, 56(1), 111-4. doi: 10.1016/00319384(94)90267-4
Da Silva Costa-Aze, V., Quiedeville, A., Boulouard, M., \& Dauphin, F. (2012). 5-HT6 receptor blockade differentially affects scopolamine-induced deficits of working memory, recognition memory and aversive learning in mice. Psychopharmacology, 222(1), 99-115. doi: 10.1007/s00213-011-2627-3

Dash, P. K., Moore, A. N., Kobori, N., \& Runyan, J. D. (2007) Molecular activity underlying working memory. Learning Eamp; Memory, 14(8), 554-63. doi: 10.1101/lm.558707

Ebert, \& Kirch. (1998). Scopolamine model of dementia: Electroencephalogram findings and cognitive performance European Journal of Clinical Investigation, 28(11), 944-9. doi: 10.1046/j.1365-2362.1998.00393.x

Ellman, G. L., Courtney, K. D., Andres, V., \& Featherstone, R. M (1961). A new and rapid colorimetric determination of acetylcholinesterase activity. Biochemical Pharmacology, 7(2), 88-95. doi: 10.1016/0006-2952(61)90145-9

Falsafi, S. K., Deli, A., Höger, H., Pollak, A., \& Lubec, G. (2012) Scopolamine administration modulates muscarinic, nicotinic and NMDA receptor systems. PLoS ONE, 7(2), e32082. doi: 10.1371/journal.pone.0032082

Fan, Y., Hu, J., Li, J., Yang, Z., Xin, X., Wang, J., et al. (2005). Effect of acidic oligosaccharide sugar chain on scopolamine-induced memory impairment in rats and its related mechanisms. Neuroscience Letters, 374(3), 222-6. doi:10.1016/j.neulet.2004.10.063

Fillit, H., Ding, W., Buee, L., Kalman, J., Altstiel, L., Lawlor, B., \& Wolf-Klein, G. (1991). Elevated circulating tumor necrosis factor levels in Alzheimer's disease. Neuroscience Letters, 129(2), 318-20. doi: 10.1016/0304-3940(91)90490-k

Gutierres, J. M., Carvalho, F. B., Schetinger, M. R. C., Agostinho, P., Marisco, P. C., Vieira, J. M., et al. (2014). Neuroprotective effect of anthocyanins on acetylcholinesterase activity and attenuation of scopolamine-induced amnesia in rats. International Journal of Developmental Neuroscience, 33, 88-97. doi: 10.1016/j.ijdevneu.2013.12.006

Harrison, F. E., Hosseini, A. H., Dawes, S. M., Weaver, S., \& May, J. M. (2009). Ascorbic acid attenuates scopolamine-induced spatial learning deficits in the water maze. Behavioural Brain Research, 205(2), 550-8. doi: 10.1016/j.bbr.2009.08.017

Härtl, R., Gleinich, A., \& Zimmermann, M. (2011). Dramatic increase in readthrough acetylcholinesterase in a cellular model of oxidative stress. Journal of Neurochemistry, 116(6), 1088-96. doi: 10.1111/j.1471-4159.2010.07164.x

Hauss Wegrzyniak, B., Lynch, M. A., Vraniak, P. D., \& Wenk, G. L. (2002). Chronic brain inflammation results in cell lss in the entorhinal cortex and impaired LTP in perforant path-granule cell synapses. Experimental Neurology, 176(2), 336-41. doi: 10.1006/exnr.2002.7966

Hritcu, L., Stefan, M., Brandsch, R., \& Mihasan, M. (2015) Enhanced behavioral response by decreasing brain oxidative stress to 6-hydroxy-1-nicotine in Alzheimer's disease rat model. Neuroscience Letters, 591, 41-7. doi: 10.1016/j.neulet.2015.02.014

Jahanshahi, M., Nickmahzar, E. G., \& Babakordi, F. (2013). The effect of Ginkgo biloba extract on scopolamine-induced apoptosis in the hippocampus of rats. Anatomical Science International, 88(4), 217-22. doi: 10.1007/s12565-013-0188-8

Jang, Y. J., Kim, J., Shim, J., Kim, C. Y., Jang, J. H., Lee, K. W., \& Lee, H. J. (2013). Decaffeinated coffee prevents scopolamine- 
induced memory impairment in rats. Behavioural Brain Research, 245, 113-9. doi: 10.1016/j.bbr.2013.02.003

Klinkenberg, I., \& Blokland, A. (2010). The validity of scopolamine as a pharmacological model for cognitive impairment: A review of animal behavioral studies. Neuroscience $\mathcal{E}$ Biobehavioral Reviews, 34(8), 1307-50. doi: 10.1016/j.neubiorev.2010.04.001

Mahmoodi, G., Ahmadi, S., pourmotabbed, A., Oryan, S., \& Zarrindast, M. R. (2010). Inhibitory avoidance memory deficit induced by scopolamine: Interaction of cholinergic and glutamatergic systems in the ventral tegmental area. Neurobiology of Learning and Memory, 94(1), 83-90. doi: 10.1016/j. nlm.2010.04.004

Marino, M. J., Rouse, S. T., Levey, A. I., Potter, L. T., \& Conn, P. J. (1998). Activation of the genetically defined $\mathrm{m} 1$ muscarinic receptor potentiates N-methyl-D-aspartate (NMDA) receptor currents in hippocampal pyramidal cells. Proceedings of the National Academy of Sciences, 95(19), 11465-70. doi: 10.1073/ pnas.95.19.11465

Mhatre, M., Floyd, R. A., \& Hensley, K. (2004). Oxidative stress and neuroinflammation in Alzheimer's disease and amyotrophic lateral sclerosis: Common links and potential therapeutic targets. Journal of Alzheimer's Disease, 6(2), 147-57. doi: 10.3233/jad-2004-6206

Perry, E. K. (1986). The cholinergic hypothesis - ten years on. British Medical Bulletin, 42(1), 63-9. doi: 10.1093/oxfordjournals.bmb.a072100

Sambeth, A., Riedel, W. J., Smits, L. T., \& Blokland, A. (2007). Cholinergic drugs affect novel object recognition in rats: Relation with hippocampal EEG. European Journal of Pharmacology 572(2-3), 151-9. doi: 10.1016/j.ejphar.2007.06.018

Soodi, M., Naghdi, N., Hajimehdipoor, H., Choopani, S., \& Sahraei, E. (2014). Memory-improving activity of Melissa officinalis extract in naïve and scopolamine-treated rats. Research in Pharmaceutical Sciences, 9(2), 107-14. PMCID: PMC4311288

Soodi, M., Saeidnia, S., Sharifzadeh, M., Hajimehdipoor, H., Dashti, A., Sepand, M. R., \& Moradi, S. (2015). Satureja bachtiarica ameliorate beta-amyloid induced memory impairment, oxidative stress and cholinergic deficit in animal model of Alzheimer's disease. Metabolic Brain Disease, 31(2), 395-404. doi: 10.1007/s11011-015-9773-y

Uma, G., \& Maheswari, S. U. (2014). Neuroprotective effects of polyherbal formulation (Indian) on noni scopolamineinduced memory impairment in mice. Internation al Journal of Pharmacy and Pharmaceutical Sciences, 6(1), 354-7. 